\title{
Metateoría sobre la juventud en dificultad social. Transición a la vida adulta
}

\section{Metatheory on youth in social distress. Transition to adulthood}

\author{
Ana Fernández-García ${ }^{1}$ \\ Universidad Nacional de Educación a Distancia, España
}

\begin{abstract}
Resumen. La investigación que se presenta analiza la transición de los jóvenes a la vida adulta. Tras la lectura de documentos especializados, se evidencia que esta evolución es muy complicada, incierta e indefinida. Más aún, para los jóvenes que presentan un cúmulo de problemáticas sociales persistentes, múltiples desventajas y vulnerabilidad social. Respecto a la metodología, se ha realizado un análisis metateórico sobre artículos de investigación especializados en el tema ubicados en revistas científicas. Los estudios analizados ponen de relieve que el concepto de juventud se suele descomponer en tres aspectos básicos que caracterizan a la vida adulta: dejar de convivir con los padres y establecer un hogar propio, conseguir un empleo estable que conlleve a la independencia económica y crear un núcleo familiar con pareja e hijos. La juventud es una etapa crucial en la vida de toda persona, un momento clave en la construcción de una identidad laboral y personal. La mediación educativa constituye un soporte esencial para fortalecer y apoyar en este tránsito a los jóvenes más vulnerables. Ante ello, las administraciones y los gobiernos deben fortalecer medidas de apoyo a estos jóvenes. Ninguna sociedad puede prescindir de los jóvenes si desea construir una comunidad cohesionada con la finalidad de fortalecer el necesario reemplazo generacional.
\end{abstract}

Palabras clave. Juventud, dificultad social, transición, investigación, metateoría.

\begin{abstract}
The presented research analyzes the transition from youth to adulthood. After reading specialized documents, it is evident that this transition is complicated, uncertain and indefinite, even more so for young people who have a heap of persistent social problems, multiple handicaps and social vulnerability. Regarding methodology, a metatheoretical analysis was carried out using specialized research articles on the subject found in scientific journals. Studies show that the concept of youth is usually broken down into three basic aspects: stop living with parents and establish own home, getting a stable job that leads to financial independence, and forming a family with partners and children; three aspects which characterize adult life. Youth is a crucial stage in the life of every person, a key moment in the construction of a personal and work related identity. Educational mediation is an essential tool to strengthen and support the most vulnerable in this transition. In response, administrations and governments must strengthen measures to support the young. No society can do without youngsters if they want to build a cohesive community in order to strengthen the necessary generational replacement.
\end{abstract}

Keywords. Youth, social difficulty, transition, research, metatheory. 


\section{Introducción}

La integración en la sociedad y en el entorno en el que se vive es un elemento esencial para la vida de cada persona, puesto que determina en gran medida la óptima convivencia y el pleno desarrollo de todas las capacidades humanas.

En la juventud, la transición a la vida adulta es un elemento clave para el logro de dicha integración. En este sentido Fernández Tilve y Malvar Méndez (2011, p. 103) afirman que "los/ las adolescentes viven ligados, inexorablemente, a etapas de transición (académicas, laborales, biológicas...), los sentimientos que la acompañan son de temor ante la posibilidad de no estar preparados para afrontar con solvencia estos cambios, de desosiego ante lo desconocido". De ahí que la transición a la vida adulta y activa adquiera especial relevancia en esta etapa vital.

En este trabajo se analiza la transición a la vida adulta de jóvenes, especialmente vulnerables. Estos jóvenes se caracterizan por la presencia en sus vidas de algunos de los siguientes rasgos: fracaso y abandono escolar temprano con las consecuentes bajas cualificaciones que les incapacita para acceder al mercado laboral; desmotivación, estereotipos y falta de confianza respecto a los estudios y al empleo; dificultades económicas; desestructuración familiar, situaciones de violencia en el hogar; problemas de salud, tanto física como psicológica; desempleo generacional familiar; violencia y criminalidad; trayectoria de varios empleos en diferentes sectores y sin continuidad; desconocimiento de las herramientas facilitadoras de empleo; barrios vecinales marginados $\mathrm{y}$ amistades peligrosas, entre otros.

El objetivo que se pretende con esta investigación es analizar los aspectos más relevantes que intervienen en la transición a la vida adulta de los jóvenes, especialmente, aquellos que sufren y conviven con alguna problemática social.

\section{Metodología}

El presente artículo se centra en la revisión y valoración de investigaciones que han estudiado la transición a la vida adulta de los jóvenes con problemática social. La metodología utilizada ha consistido en el análisis metateórico de dichas investigaciones, publicadas en artículos científicos.

Para Cortés del Moral (2000, p. 361), la metateoría es "un campo disciplinar que se ocupa del estudio de la teoría, es decir, de los saberes acumulados en un área particular de conocimiento científico o humanístico". Este procedimiento metodológico facilita reducir la información hallada agrupándola en categorías y reduce la probabilidad de que los resultados de las diferentes búsquedas puedan atribuirse al azar al obtener una panorámica global de las tendencias surgidas. Esta metodología se ha aplicado a un conjunto de investigaciones que estudian la misma problemática (tránsito a la vida adulta) y que comparten las principales variables dependientes e independientes. El proceso sistemático llevado a cabo en este análisis metateórico consta de tres fases que se relatan a continuación:

La primera fase ha consistido en la definición de las dimensiones más importantes que caracterizan el tránsito a la vida adulta de los jóvenes en dificultad social (definición, características, problemáticas...). Para ello, se han consultado bases de datos especializadas que contienen revistas científicas, con el propósito de seleccionar las investigaciones objeto de estudio, a saber: Dialnet; Eric; Isoc y Redalyc. Por su parte, para la elección de dichas revistas se han definido tres requisitos de selección: a) haberse publicado entre los años 2010-2014; b) poseer una metodología cuantitativa, cualitativa o mixta y c) ser de ámbito nacional e internacional.

En segundo lugar, se ha procedido a la elección de los artículos según los siguientes criterios: su 
grado de pertinencia y suficiencia. Por medio del criterio de pertinencia se han diseñado dimensiones (conceptualización y caracterización) más pertinentes al tema objeto de estudio. Mediante el criterio de suficiencia, hemos podido escoger los artículos que se ajustan suficientemente al tema de investigación (poseen como temática central la transición a la vida adulta de jóvenes con problemática social). Han sido quince artículos los que cumplen los requisitos de selección y conforman la muestra de estudio.

Finalmente, se ha descrito la tendencia actual sobre la transición a la vida adulta de los jóvenes en dificultad social. Para ello, hemos realizado un análisis de contenido de las dimensiones seleccionadas con el propósito de descubrir la tendencia reinante en este campo de investigación.

\section{Muestra}

A continuación, se exponen los análisis descriptivos extraídos de la muestra objeto de estudio: año de publicación, base de datos, metodología y ámbito. En relación con el año de publicación, el $34 \%$ de los artículos pertenecen al 2010. El $27 \%$ de las investigaciones han sido publicadas en el año 2011 y con un $13 \%$ se encuentra el porcentaje referido para los siguientes años: 2012, 2013 y 2014, respectivamente (figura 1).

Respecto a las bases de datos bibliográficas utilizadas para la selección de los artículos, se han empleado las siguientes: Dialnet, Eric, Isoc y Redalyc. Un $20 \%$ de las investigaciones se hallan ubicadas en la base de datos Dialnet. Cabe destacar que el $86,7 \%$ de los artículos se encuentran ubicados en esta base de datos. El 13,3\% de los artículos se hallan en Eric. El resto de los artículos se encuentran en varias bases de datos, tal como puede comprobarse en la figura 2.

En relación con la metodología, el 60 \% de los artículos son de carácter cualitativo y el $40 \%$ han empleado una metodología mixta. Como puede

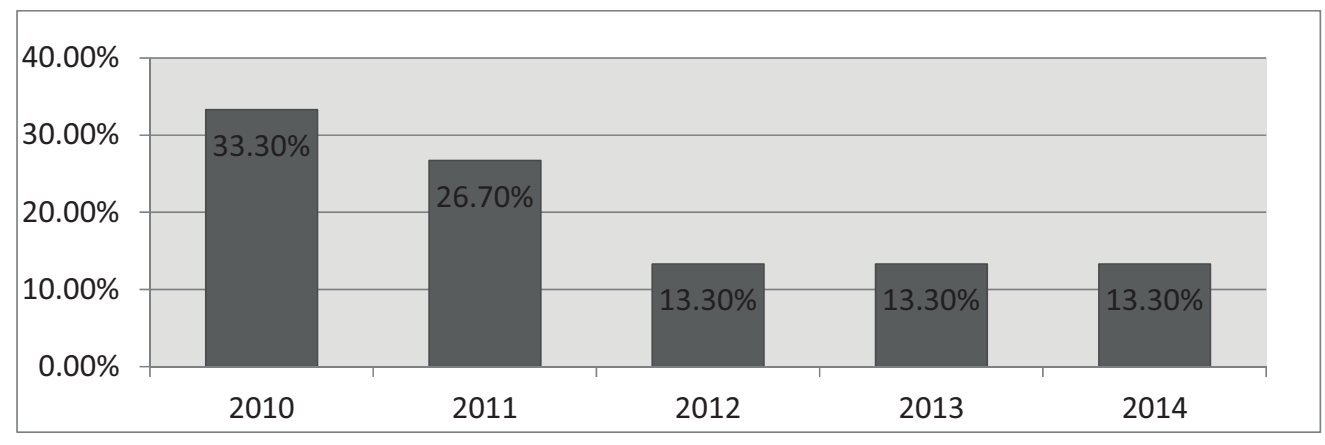

Figura 1. Año de publicación. Nota. Elaboración propia.

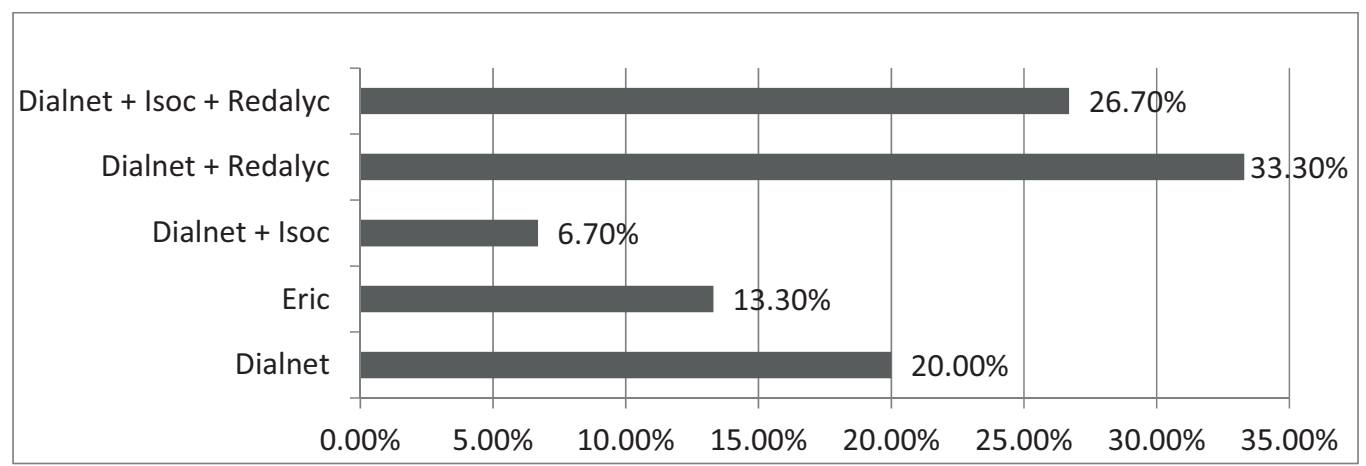

Figura 2. Bases de datos. Nota. Elaboración propia. 
apreciarse, ningún artículo presenta una metodología cuantitativa en exclusiva.

Por último, y según el ámbito de estudio, el $40 \%$ de las investigaciones son nacionales (España), frente al $60 \%$ de los artículos que son internacionales (Colombia, México, Chile, Brasil, Canadá y La India) (figura 3).

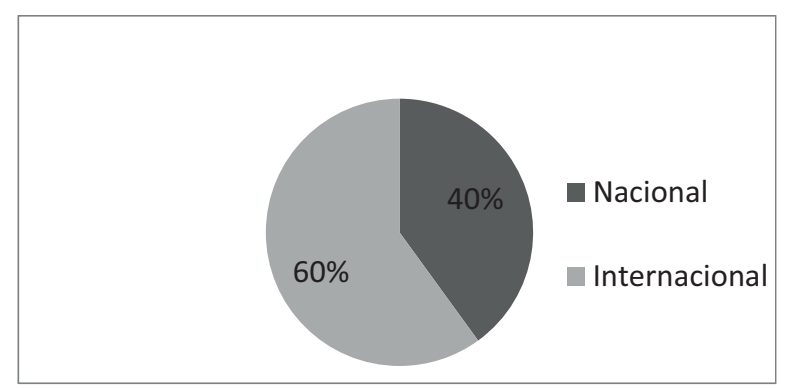

Figura 3. Ámbito. Nota. Elaboración propia.

\section{Resultados}

Melo Vieira y Miret Gamundi (2010, p. 77) definen la transición a la vida adulta como "el cambio desde un estado social caracterizado por la dependencia y por la necesidad de soporte y apoyo, propio de la condición infantil, a una nueva condición de individuo completamente emancipado y autónomo, propio de la condición adulta". Este cambio afecta tanto a la dimensión familiar como a la dimensión ocupacional de los sujetos.

Para Otero (2010, p. 4), que ha investigado la transición a la vida adulta de los jóvenes en América Latina, esta se define como "un proceso hacia la independencia respecto del hogar de origen, tomando como punto de partida la coexistencia de distintos modos de desarrollo de las mismas (sic)". Se otorga un carácter abierto a las distintas transiciones que puede experimentar la juventud.

Por su parte, Parrilla Latas, Gallego Vega y Moriña Díez (2010, p. 215) indican que dicha transición puede ser definida como "el proceso por el que los jóvenes acceden a la independen- cia económica, normalmente a través del empleo, y a la formación, y constitución de un hogar independiente".

Tinoco Ponciano y Féres-Carneiro (2012, p. 219) argumentan que "a partir de la adolescencia, hay un período de experimentación caracterizado por el aplazamiento de la entrada definitiva en el mundo adulto, en el que los padres siguen responsables de sus hijos, nombrándose ese período de transición para la vida adulta. La vida adulta puede ser aplazada indefinidamente debido a la variedad de posibles caminos que los jóvenes encuentran".

La transición de los jóvenes a la vida adulta es complicada, incierta e indefinida, más aun para aquellos jóvenes que presentan un cúmulo de problemáticas sociales persistentes, múltiples desventajas y vulnerabilidad social (Fernández Tilve y Malvar Méndez, 2011; Juárez y Gayer, 2014; Melo Vieira y Miret Gamundi, 2010; Morrow, 2013; Parrilla Latas, Gallego Vega y Moriña Díez, 2010; Otero, 2010; Tinoco Ponciano y Féres-Carneiro, 2012). Un estudio llevado a cabo en Canadá por Goyette (2010, p. 43) revela que “el tránsito a la vida adulta en los jóvenes atendidos desde los servicios sociales es muy complicado, ya que estos jóvenes acumulan más dificultades psicosociales y rupturas con el mundo familiar. Paradójicamente, están confrontados más a menudo con la exigencia de autonomía y de independencia".

Otero (2010) sostiene que las transiciones de la juventud a la edad adulta, ya no son lineales (educación para el empleo), sino sincrónicas (la educación y el empleo suceden al mismo tiempo) o reversibles, es decir, los movimientos "yoyo" (de la educación al empleo, y de vuelta a la educación por falta de empleo, reconsiderando un aumento de estudios y/o reciclaje profesional). Dicho aspecto se corrobora en otras investigaciones (Dávila y Ghiardo, 2012; Goyette, 2010; Morrow, 2013) al definir tales procesos progresi- 
vos como tipo yo-yo, reversibles o laberínticos; es decir, "las experiencias de vida y de transición de muchos sujetos jóvenes son más accidentadas, menos previsibles, más extensas y más fracturadas, lo que se ha traducido en un incremento de situaciones y experiencias de mayor desigualdad, mayor vulnerabilidad individual y una tendencia al incremento de la polarización y de la exclusión de estos sectores de la sociedad" (Rivera-González, 2011, p. 343).

Las sociedades son cada vez más imprevisibles, con una gran complejidad social y la incertidumbre está presente en muchas esferas de la vida, lo que en ocasiones el paso a la vida adulta puede resultar angustioso o desafiante. Otero (2010, p. 2) afirma que "en el panorama latinoamericano actual, se corrobora la presencia de estructuras sociales cada vez más desiguales, heterogéneas y sujetas al avance de dinámicas devastadoras".

Cabe resaltar que las transiciones han cambiado a lo largo de las diferentes generaciones, porque no existen expectativas de transición estandarizadas para la mayoría de los jóvenes dentro de un mismo territorio. El mundo globalizado en el que estamos inmersos estimula nuevos estilos de vida más allá de las fronteras, aunque "este proceso de globalización podría haber llevado a una mayor homogeneización de las transiciones a escala mundial y, sin embargo, dio por resultado una mayor diversificación de trayectorias" (Juárez y Gayer, 2014, p. 2).

Un aspecto muy relevante ha sido resaltado por Tinoco Ponciano y Féres-Carneiro (2012) que han realizado un estudio en Río de Janeiro, donde se explicita que las experiencias individuales y sociales de los jóvenes se conectan directamente a su familia y a su red social. Por ello, afirman que "los comportamientos transicionales para la vida adulta tienen el potencial de influir en la vida de los otros miembros de la familia, especialmente la de los padres" (p. 220).
En este periodo de transición a la vida adulta, los padres siguen siendo responsables de sus hijos. Pero, de acuerdo con esta dependencia, los padres necesitan crear condiciones para el desarrollo adulto de los hijos al cumplir el rol de ofrecer apoyo para el crecimiento y la autonomía de su descendencia. Marzana, Pérez-Acosta, Marta y González (2010) narran cómo los jóvenes participantes de su estudio (cuarenta adultos jóvenes colombianos) han señalado como elemento esencial el apoyo (elemento central de la trasmisión familiar) que le ofrecían sus padres. Ello se entiende tanto en términos materiales (sostenimiento económico) como emocionales. El primero atribuido más a la figura paterna y el segundo, a la materna.

Tinoco Ponciano y Féres-Carneiro (2012, p. 221) reiteran que "los padres siguen siendo una importante fuente de apego seguro, trabajando el equilibrio continuado entre la conexión y el aumento de la autonomía"; sin embargo, advierten que en muchas ocasiones los padres no saben qué hacer para ayudar a que sus hijos crezcan $\mathrm{y}$ en ocasiones se quedan perplejos ante dicho aplazamiento. No obstante, también puede suceder lo contrario, puesto que en la investigación llevada a cabo por Marzana, Pérez-Acosta, Marta y González (2010), algunos de los jóvenes que fueron entrevistados indicaron una falta de independencia y libertad personal debido a la tendencia al "hipercontrol" por parte de los padres.

Una vez analizadas las investigaciones, se puede concluir que el término juventud se suele descomponer en tres aspectos básicos: dejar de convivir con los padres y fundar un hogar independiente (Dávila y Ghiardo, 2012; Melo Vieira y Miret Gamundi, 2010; Otero, 2010; Parrilla Latas, Gallego Vega y Moriña Díez, 2010; Rivera-González, 2011), conseguir un trabajo estable que abra la puerta a la independencia financiera (Marzana, Pérez-Acosta, Marta y González, 2010; Melo Vieira y Miret Gamundi, 2010; Otero, 
2010; Parrilla Latas, Gallego Vega y Moriña Díez, 2010; Rivera-González, 2011; Tinoco Ponciano y Féres-Carneiro, 2012) y formar un núcleo familiar con pareja e hijos (Dávila y Ghiardo, 2012; Marzana, Pérez-Acosta, Marta y González, 2010; Melo Vieira y Miret Gamundi, 2010; Otero, 2010; Rivera-González, 2011; Tinoco Ponciano y Féres-Carneiro, 2012). Dichos tres aspectos caracterizan a la vida adulta.

Pero, los jóvenes que tienen que vivir esta etapa en contextos de crisis se encuentran con muchos riegos y dificultades. Dávila y Ghiardo (2012, p. 74) que han investigado en el contexto de Chile, afirman que "los cambios en el sistema de trabajo han impuesto formas cada vez más inestables y flexibles de inserción laboral que sin duda repercuten en los procesos de conformación de independencias económicas y familiares".

La literatura científica muestra con claridad cuáles son las principales dificultades para la integración de esta población juvenil especialmente vulnerable. En general, son pocos los que acaban sus estudios secundarios (Goyette, 2010; Rivera-González, 2011). Lo más frecuente es que los empleos ocupados sean poco remunerados y gratificantes (Benedicto y Morán, 2014; Dávila y Ghiardo, 2012; Goyette, 2010) y una gran mayoría de ellos vive bajo el umbral de la pobreza (Benedicto y Morán, 2014; Goyette, 2010). También, es importante resaltar que los jóvenes con antecedentes de acogimiento familiar recurren con más frecuencia a los servicios y las ayudas sociales que la población general.

Estos jóvenes ven su futuro arduo y difícil, puesto que como se expone en diversas investigaciones (López, Santos, Bravo y F. del Valle, 2013; Otero, 2010) los jóvenes, especialmente con graves dificultades, afirman que en corto plazo no ven probable alcanzar la independencia familiar, tanto en lo que se refiere a lo económico como a la vivienda. Rotundamente, no contem- plan o dudan significativamente ante la posibilidad de irse de sus hogares, puesto que como bien indica Rivera-González (2011, p. 333) “estas transiciones y estos nuevos roles son cada vez más difíciles de asumir", para una juventud carente de oportunidades.

La situación de la mujer es otro eje destacado en las investigaciones analizadas. En América Latina, en el estudio llevado a cabo por Otero (2010), ha encontrado que aquellas mujeres que desean acceder a un empleo, no implica que desestimen la maternidad. Estas jóvenes desean un futuro profesional en activo y ser madres en simultáneo. Desde Canadá, Goyette (2010, p. 51) afirma que "la maternidad influye en las jóvenes, ya que por un lado la maternidad adolescente les parece una oportunidad de liberación y reconocimiento social, pero en otras ocasiones esta situación les conduce a situarlas en una dinámica relacional basada en una distribución tradicional de roles sociales, donde el hombre es el proveedor". En el contexto de Chile, Dávila y Ghiardo (2012, p. 74) relatan que "en relación con la fecundidad, existe una tendencia general que apunta a la postergación del nacimiento del primer hijo; [...] lo que se expresa también en la edad promedio en que se contrae matrimonio, que también va en aumento".

Una vez estudiadas las investigaciones más destacables sobre el tema, se puede afirmar que han surgido nuevas formas de identidad femenina, que abogan por una independencia económica y una autonomía en sus relaciones afectivas, aunque se da importancia a la creación de una familia propia.

El tránsito a la vida adulta está asociado a la prolongación general de la etapa juvenil. Goyette (2010, p. 44) concluye que "esa prolongación conduce a que esta juventud sea menos independiente o autosuficiente comparada con la juventud de las generaciones anteriores, por causa de que los jóvenes viven más tiempo en casa de sus 
padres y de que estos contribuyen a su subsistencia por un tiempo más largo, particularmente durante el período en que los jóvenes estudian". No obstante esta apreciación, puede ser considerada por otros autores positivamente como por ejemplo Melo Vieira y Miret Gamundi (2010, p. 103) cuando afirman "que el retraso en la entrada en la edad adulta, o la ganancia en tiempo de juventud - todo depende de la perspectiva-, es proporcional al aumento de la esperanza de vida en España". Estos autores han investigado determinados países (España, Italia...) que presentan transiciones más tardías, donde existen fuertes lazos familiares (económicos y culturales) y se comparte responsabilidad y trabajo. Se caracteriza por una estructura basada en la solidaridad e interdependencia, marcadas por asimetrías de género y de generación.

Respecto a las relaciones interpersonales, los jóvenes pueden moverse en redes que les den soporte (tal como la familia y/o la pareja) o redes inhibitorias que los hacen aún más vulnerables (amigos perjudiciales) (Goyette, 2010). Por ello, "potenciar las redes sociales naturales de apoyo se convierte en una prioridad a la hora de diseñar intervenciones de ayuda a la transición a la vida activa" (Parrilla Latas, Gallego Vega y Moriña Díez, 2010, p. 228).

Por supuesto, es imprescindible el compromiso del joven con el proceso de intervención (Goyette, 2010). En la investigación llevada a cabo por Melendro Estefanía (2011, p. 339), se observó que "entre el $65,5 \%$ y el $82,4 \%$ de los jóvenes mantienen aún relación con personas que conocieron en los programas de inserción sociolaboral o en los centros de protección de menores, tanto educadores como antiguos compañeros, que en algún caso se han convertido en amigos cercanos, parejas o compañeros de trabajo", Al aludir la importancia de crear una buena red de confianza.
Hay que tener en cuenta que en el tránsito a la vida adulta persisten dinámicas de desigualdad social, determinadas por la desigual distribución de oportunidades y riesgos sociales (Otero, 2010); diferentes niveles socioeconómicos, de género y familiar (Dávila y Ghiardo, 2012); así como el reconocimiento de la influencia de determinantes externos macro como la sociedad, la cultura y la historia (Marzana, Pérez-Acosta, Marta y González, 2010).

Para ayudar a los jóvenes, especialmente los más vulnerables, en la transición a la vida adulta, diversos autores han expuesto sus propuestas a fin de ofrecer a la comunidad educativa las claves para el éxito de la intervención. Vega Fuente y Arimendi Jáuregui (2011) defienden la necesidad de asumir los procesos de transición a la vida adulta en los jóvenes, con una mirada global y amplia, para concretar la mediación educativa mediante acciones emanadas y desarrolladas desde las situaciones singulares de cada individuo en las comunidades locales (centros formativos, familias, barrios, etc.).

En este sentido, Goyette (2010, p. 44) aclara que "la inserción de los jóvenes ya no se realiza según un modelo social prácticamente unificado, sino que deja lugar a una cierta individualización de los recorridos, compuesto de avances $y$ retrocesos en las diversas transiciones profesionales, residenciales y familiares".

Además, Vega Fuente y Arimendi Jáuregui (2011, p.215) reiteran que "la mediación es una excelente herramienta para la comunicación, la tolerancia, la práctica democrática y de formación". Asimismo, "los hace más responsables y contribuye a enriquecer la ciudadanía, creando lazos de convivencia y de solidaridad como de formación escolar y profesional".

En definitiva, se debe ayudar a los jóvenes para que asuman un papel activo en la transición a la vida adulta en condiciones de conocimiento, 
motivación y plena responsabilidad. Solo así, los adolescentes tendrán la capacidad para emprender su propio proyecto personal de manera positiva acorde con sus intereses y circunstancias. “De los apoyos y recursos con que cuenten, de las posibilidades y expectativas sobre la misma podrá derivarse o no la salida de la trayectoria excluyente, pudiendo convertirse por tanto el tránsito a la vida adulta en una posibilidad de mejora, de cambio" (Parrilla Latas, Gallego Vega y Moriña Díez, 2010, p. 213).

\section{Conclusiones}

La juventud es una etapa muy relevante en la vida de cada persona. Es una fase inicial y preparatoria para la transición que se experimenta entre la niñez y la adultez, en la cual se busca ganar autonomía e independencia. Estos son momentos muy trascendentales en la vida de cualquier persona, porque contribuyen a la construcción de una identidad personal y de una identidad laboral.

A nivel mundial, se asiste a un movimiento de protesta que guarda relación con la precaria situación económica y laboral que sufren estos jóvenes. El futuro que tienen por delante se presume difícil y, en ocasiones, desalentador, pues existen muchas situaciones de desigualdad. Asimismo, luchan contra etiquetas que les otorgan tales como "NiNi", "generación perdida" ... centrando el foco del problema en ellos mismos.

Sin embargo, hoy, en cualquier sociedad del mundo, la transición de los jóvenes a la vida adulta es muy complicada, incierta e indefinida, más aún para los jóvenes que presentan un cúmulo de problemáticas sociales persistentes, múltiples desventajas y vulnerabilidad social.

Las transiciones ya no son lineales, porque los jóvenes en primer lugar van a la escuela y adquieren una formación para acceder a un puesto de trabajo (educación para el empleo); sino sincrónicas (situaciones simultáneas que combinan la educación y el empleo) o reversibles, es decir, los movimientos "yo-yo" (vuelta a la educación después de la pérdida de un empleo como medio para la adquisición de formación, o reciclaje profesional que permita de nuevo encontrar un puesto de trabajo).

Ante ello, las administraciones gubernamentales deben ofrecer oportunidades de formación continua e inserción en el mercado laboral. La crisis económica mundial agudiza la precaria situación de estos jóvenes, porque muchos de los recortes presupuestarios realizados por los gobiernos afectan a los sectores de la población con menos recursos.

Todavía queda mucho por hacer, sobretodo en la asunción de nuevos desafíos (por parte de diversos organismos y administraciones) para el logro de una efectiva inserción social y laboral. De esta forma, "los [jóvenes] que ocupan un empleo o los que estudian (o ambas cosas simultáneamente), se insertan más a menudo en una trayectoria constructiva" (Goyette, 2010, p. 50) y se alejan de formas de vida que conducen a la exclusión y marginación social.

Ninguna sociedad puede prescindir de los jóvenes si desea construir una comunidad cohesionada e integradora con la finalidad de fortalecer el necesario reemplazo generacional.

\section{Referencias}

Benedicto, J. y Morán, M. L. (2014). ¿Otra clase de politización? Representaciones de la vida colectiva y procesos de implicación cívica de los jóvenes en situación de desventaja. Revista Internacional de Sociología, 72(2), 429-452.

Cortes del Moral, R. (2000). La filosofía y la racionalidad contemporánea. Guanajuato: Universidad de Guanajuato. 
Dávila, O. y Ghiardo, F. (2012). Transiciones a la vida adulta: generaciones y cambio social en Chile. Última década, 20(37), 69-83.

Fernández Tilve, M. D. y Malvar Méndez, M. L. (2011). El papel de la escuela en la transición a la vida activa del/la adolescente: buscando buenas prácticas de inclusión social. Revista de Formación e Innovación Educativa Universitaria, 4(2), 101-114.

Goyette, M. (2010). El tránsito a la vida adulta de los jóvenes atendidos desde los Servicios Sociales. Pedagogía social. Revista interuniversitaria, 17, 43-56.

Juárez, F. y Gayet, C. (2014). Transiciones a la Vida Adulta en Países en Desarrollo. Annual Review of Sociology, 40(1), 1-18.

López, M., Santos, I., Bravo, A. y F. Del Valle, J. (2013). El proceso de transición a la vida adulta de jóvenes acogidos en el sistema de protección infantil. Anales de Psicología, 29(1), 187-196.

Marzana, D., Pérez-Acosta, A. M., Marta, E. y González, M. I. (2010). La transición a la edad adulta en Colombia: una lectura relacional. Avances en psicología latinoamericana, 28(1), 99-112.

Melendro Estefanía, M. (2011). El tránsito a la vida adulta de los jóvenes en dificultad social: la incidencia de la intervención socioeducativa y la perspectiva de profesionales y empresarios. Revista de educación, 356, 327-352.
Melo Vieira, J. y Miret Gamundi, P. (2010). Transición a la vida adulta en España: una comparación en el tiempo y en el territorio utilizando el análisis de entropía. Revista Española de investigaciones sociológicas, 131(1), 75-107.

Morrow, V. (2013). Troubling transitions? Young people's experiences of growing up in poverty in rural Andhra Pradesh, India. Journal of Youth Studies, 16(1), 86-100.

Otero, A. E. (2010). Los avatares de la transición a la vida adulta, el papel de la educación y el trabajo en los recorridos juveniles. Margen, 59, 1-13.

Parrilla, A., Gallego, C. y Moriña, A. (2010). El complicado tránsito a la vida activa de jóvenes en riesgo de exclusión: una perspectiva biográfica. Revista de educación, 351, 211-233.

Rivera-González, J. G. (2011). Tres miradas a la experiencia de la exclusión en las juventudes en América Latina. Revista Latinoamericana de Ciencias Sociales, Niñez y Juventud, 1(9), 331-346.

Tinoco Ponciano, E. L. y Féres-Carneiro, T. (2013). Transición para la vida adulta: la transformación del rol parental. Interamerican Journal of Psychology, 46(2), 219-228.

Vega Fuente, A. y Aramendi Jáuregui, P. (2011). La mediación educativa de los programas de cualificación profesional inicial: a propósito de las drogas. Educación XX1, 14(2), 213-236.

Recibido: 5 de julio de 2016 Aceptado: 29 de setiembre de 2016 